\title{
A review on spirulina (Arthrospira Platensis) for its antioxidant and neuroprotective effect
}

\author{
Rashmi B.R
}

Lecturer, Dept, of Physiology, A.J. Institute of Allied Health Sciences, Kuntikana, Mangalore, Karnataka, India

*Corresponding Author: Rashmi B.R

Email: bhaabay2003@yahoo.co.in

\begin{abstract}
Spirulina (Arthrospira platensis), belongs to a class of cyanobacteria. It is a free-floating filamentous microalgae and is also capable of photosynthesis. The nutritional content of Spirulina reveals high content of protein (60-70\% by dry weight), vitamins, minerals, essential fatty acids, and other nutrients. The Food and Agriculture Organization of the United Nations (FAO) position paper describes Arthrospira platensis as follows: "An easily digestible high protein product with high levels of beta-carotene, vitamin $B_{12}$, iron and trace minerals, and the rare essential fatty acid $\gamma$-linolenic acid also called gammalinolenic acid (GLA), or omega-6". Arthrospira platensis has been generally recognized as safe (GRAS) for human consumption. Human clinical studies and animal studies over the past several decades support such notion. Also, FDA had no question on the GRAS notice of Arthrospira platensis.
\end{abstract}

Keywords: Arthrospira Platensis.

\section{Introduction}

Spirulina (Arthrospira platensis) belongs to family of blue-green algae. They have characteristic spiral shape in their morphology apperence. ${ }^{1}$ It is free-floating filamentous microalgae growing in alkaline water bodies. $^{2}$

Arthrospira platensis is considered under phylum of Cyanobacteria. It has been given importance as a popular food and nutritional supplements. This Cyanobacterium exists as either blue-green bacteria or blue-green algae in nature. Few thousands of years ago, certain African, Asian, and Mexican civilizations began to explore spirulina's beneficial medicinal properties, thus introducing its importance to the world. ${ }^{3}$

This alga represents an important staple diet in humans and has been used as a source of protein and vitamin supplement in humans without any significant side-effects. It has high (up to 70\%) content of protein, vitamins, especially $\mathrm{B}_{12}$ and provitamin $\mathrm{A}$ ( $\beta$ carotenes), and also minerals, especially iron. It has been found rich in phenolic acids, tocopherols and $\gamma$ linolenic acid. It can be easily digested as it lacks cellulose cell walls. ${ }^{4}$

Because of its apparent ability to stimulate whole human physiology, Spirulina exhibits therapeutic functions such as antioxidant, antibacterial, antiviral, anticancer, anti-inflammatory, anti-allergic and antidiabetic and plethora of beneficial functions. ${ }^{5}$

Toxicological studies on Arthrospira platensis have proven Spirulina's safety. Spirulina now belongs to the substances that are listed by the US Food and Drug Administration under the category Generally Recognized as Safe (GRAS). ${ }^{6-10}$

C-phycocyanin (C-PC) is one of the major biliproteins of Spirulina with antioxidant and radical scavenging properties. C-PC, a selective cyclooxygenase-2 inhibitor, induces apoptosis in lipopolysaccharide-stimulated RAW 264.7 macrophages. It is also known to exhibit antiinflammatory and anticancer properties. ${ }^{11}$

Previous studies reveal that iron is one of the most important agents that produce oxidative stress and decline of neuronal functions. Protean extract and phycocyanin of Arthrospira platensis exerted antioxidant property by protecting the activity of the cellular antioxidant enzymes SOD, Catalase (CAT), Glutathine peroxidase (GPx), and Glutathina reductase (GR), lipid peroxidation inhibitory activity and glutathione levels induced by iron. These results suggested that Arthrospira platensis protean extract is a powerful antioxidant through a mechanism related to 
antioxidant activity, capable of interfering with radical mediated cell death. Arthrospira platensis may also be useful in diseases known to be aggravated by reactive oxygen species and in the development of novel treatments for neurodegenerative disorders as long as iron has been implicated in the neuropathology of several neurodegenerative disorders such as Alzheimer's or Parkinson diseases. ${ }^{12}$

Neuroinflammation plays a critical role in loss of dopamine neurons during brain injury and in neurodegenerative diseases. Diets enriched in foods with antioxidant and anti-inflammatory actions may modulate this neuroinflammation. In earlier study, it was revealed that 6-hydroxydopamine (6-OHDA) injected into the dorsal striatum of normal rats, causes a progressive loss of dopamine neurons in the ventral mesencephalon with a significant increase in OX-6(MHC class II) positive microglia was found in animals fed with blueberry- and spirulina-enriched diets in both the striatum and the globus pallidus. At 1 month after the lesion, the number of OX-6-positive cells was reduced in rats fed with blueberry and spirulina-enriched diets with enhanced striatal dopamine recovery. ${ }^{13}$

The hippocampus is the focus of many studies today, since this brain structure has high zinc concentration and is highly involved in many forms of cognitive deficits as a consequence of cholinergic deficiency and has achieved prominence because of dementia in ageing and Alzheimer's disease. Thus, it is now apparent that cognitive impairment may not be attributed to a single neurotransmitter, but rather, alterations and interactions of several systems in different brain regions. In animal models of iron deficiency it is apparent that dopaminergic interaction with the opiate system and cholinergic neurotransmission may be defective. ${ }^{15}$

It is well known fact that free radicals are involved in neurodegenerative disorders, such as ischemia and aging. One of the earlier study suggest that there was reduction in the volume of infarction in the cerebral cortex and an increase in post-stroke locomotor activity, thus exhibiting neuroprotective effects in focal ischemic brain.

The alga contains a wide spectrum of prophylactic and therapeutic nutrients that include B-complex vitamins, minerals, proteins, gamma-linolenic acid and the super anti-oxidants such as beta-carotene, vitamin $\mathrm{E}$, trace elements and a number of unexplored bioactive compounds. Because of its apparent ability to stimulate whole human physiology, Spirulina exhibits therapeutic functions such as antioxidant, antibacterial, antiviral, anticancer, anti-inflammatory, anti-allergic and anti-diabetic and plethora of beneficial functions. Spirulina consumption appears to promote the growth of intestinal micro flora as well. The review discusses the potential of Spirulina in health care management. ${ }^{15}$

This study clearly indicates the possible role of Arthrospira platensis in reducing ischemia / reperfusion-induced apoptosis and cerebral infarction. ${ }^{16}$

\section{Possible role of arthrospira platensis in oxidative stress and neurodegenerative disorder \\ Depression}

Classical theory of depression states that the decrease in the neurotransmitter levels in the brain leads to the pathogenesis of clinical depression and recent studies have also shown the involvement of oxidative stress in the phenomenon. Recent evidence suggests that depression may be associated with neurodegeneration and reduced neurogenesis in the hippocampus. ${ }^{17-19}$

\section{Dementia}

Dementia (taken from Latin, originally meaning "madness", from de- "without" + ment, the root of mens "mind") is a serious loss of global cognitive ability in a previously unimpaired person, beyond what might be expected from normal aging. It may be static, the result of a unique global brain injury, or progressive, resulting in long-term decline due to damage or disease in the body. Although dementia is far more common in the geriatric population (about $5 \%$ of those over 65 are said to be involved), it can occur before the age of 65 , in which case it is termed "early onset dementia".

\section{Parkinson's disease}

Parkinson's disease (PD) is the second most common neurodegenerative disorder, after Alzheimer's disease. The typical clinical motor syndrome of PD is 
associated with neurodegeneration and neuronal loss in the substantia nigra and the presence of inclusions that contain the protein $\alpha$-synuclein ( $\alpha$-syn) known as

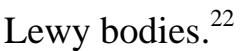

\section{Parkinson disease dementia (PDD)}

Parkinson disease dementia (PDD) can exacerbate the disabilities caused by motor symptoms in PD, and the presence of cognitive impairment or dementia in patients with PD is associated with a loss of independence, a lower quality of life, and a shorter survival time than PD patients without dementia. The course of decline in PDD is progressive over time with periods of rapid worsening. ${ }^{23,24}$

The antioxidant and/or anti-inflammatory activities of Arthrospira platensis have been demonstrated in a large number of preclinical studies. However, a limited number of clinical trials have been carried out so far to confirm such activities in human. ${ }^{25}$

\section{Conclusion}

Hence based on earlier studies on Arthrospira platensis for its antioxidant and neuroprotective effect, it is predicted that further preclinical studies on Arthrospira platensis related to depression, dementia, Parkinsonism and Parkinson disease dementia (PDD) could throws light on different angle as an adjuvant in the treatment of diseases with oxidative stress and neurodegeneration.

\section{Source of Funding}

None.

\section{Conflict of Interest}

None.

\section{References}

1. Lee RE. Phycology. 2nd ed. Cambridge: Cambridge University Press; 1989: 91.

2. Deng R, Chow TJ. Hypolipidemic, antioxidant, and antiinflammatory activities of microalgae Spirulina. Cardiovasc Ther 2010;28(4):e33-45

3. D.Jalaja Kumari, B. Babitha, SK. Jaffar, M.Guru Prasad. Ibrahim and Khan SA et al. Potential Health Benefits of Spirulina Platensis. Pharmanest 2011;2(5-6):417-22

4. J. C. Dillon, A. P. Phuc, and J. P. Dubacq, "Nutritional value of the alga Spirulina. World Rev Nutr Dietetics 1995;77:32-46.
5. Kulshreshtha A, Zacharia AJ, Jarouliya U, Bhadauriya P, Prasad GB, Bisen PS et al. Spirulina in health care management. Curr Pharm Biotechnol 2008;9(5):400-5.

6. L. M. Tarantino, "Agency Response Letter GRAS Notice No. GRN000127," FDA Home page, October 2003.

7. M. Salazar, G. Chamorro, S. Salazar, and C. Steele, "Effect of Spirulina maxima consumption on reproductive and peri- and postnatal development in rats," Food and Chemical Toxicology 1996:353-9.

8. G. Chamorro, S. Salazar, L. Favila-Castillo, C. Steele, and M. Salazar, "Reproductive and peri-and postnatal evaluation of Spirulina maxima in mice," J Appl Phycol 1997;9(2):107-12.

9. M. Salazar, E. Martínez, E. Madrigal, L. E. Ruiz, and G. A. Chamorro, "Subchronic toxicity study in mice fed Spirulina," J Ethnopharmacol 1998;62(3):235-41.

10. Belay, "The potential application of Spirulina (Arthrospira) as a nutritional and therapeutic supplement in Health management," J Am Nutraceutical Assoc 2002;5:27-48.

11. M. C. Reddy, J. Subhashini, S. V. K. Mahipal et al., "CPhycocyanin, a selective cyclooxygenase- 2 inhibitor, induces apoptosis in lipopolysaccharide-stimulated RAW 264.7 macrophages," Biochemical Biophysical Res Commun 2003;304(2):385-92.

12. Bermejo-Bescós P, Piñero-Estrada E, Villar del Fresno AM. Neuroprotection by Spirulina platensis protean extract and phycocyanin against iron-induced toxicity in SH-SY5Y neuroblastoma cells. Toxicol In Vitro 2008;22(6):1496-502.

13. Strömberg I, Gemma C, Vila J, Bickford PC. Blueberryand spirulina-enriched diets enhance striatal dopamine recovery and induce a rapid, transient microglia activation after injury of the rat nigrostriatal dopamine system. Exp Neurol 2005;196(2):298-307.

14. Wang Y, Chang CF, Chou J, Chen HL, Deng X, Harvey BK et al. Dietary supplementation with blueberries, spinach, or spirulina reduces ischemic brain damage. Exp Neurol 2005;193(1):75-84.

15. Rang HP, Dale MM, Ritter JM, and Flower RJ. Pharmacology. London: Churchill Livingstone Elsevier; 2008.

16. American Psychiatric Association. Diagnostic and Statistical Manual of Mental Disorders. Washington, DC: APA Press; 2000.

17. Sarandol A, Sarandol E, Eker SS, Erdinc S, Vatansever E, Kirli S et al. Human Psychopharmacol. Clin Exp 2007;22(2):67-73.

18. Sadock, Sadock BJ, Kaplan VA \& Sadock's concise textbook of clinical psychiatry (3rd ed.). Philadelphia: Wolters Kluwer/Lippincott Williams \& Wilkins; 2008:52.

19. Fadil H, Borazanci A, Haddou E. A. B,Yahyaoui M, Korniychuk E, Jaffe S. L, Minagar,. "Early Onset Dementia". International Review of Neurobiology. Int Rev Neurobiol 2009;84:245-62.

20. Hely MA, Reid WG, Adena MA, Halliday GM, Morris JG. The Sydney multicenter study of Parkinson's disease: the 
inevitability of dementia at 20 years. Mov Disord 2008;23:837-44.

21. Rosenthal E, Brennan L, Xie S. Association between cognition and function in patients with Parkinson disease with and without dementia. Mov Disord 2010;25(9):11706.

22. Emre M, Aarsland D, Brown R. Clinical diagnostic criteria for dementia associated with Parkinson's disease. Mov Disord 2007a;22(12):1689-707; quiz 1837.

23. D.Jalaja Kumari, B. Babitha1, SK. Jaffar, M.Guru Prasad. Ibrahim and Khan SA. Potential Health Benefits of Spirulina Platensis. Pharmanest 2011;2(5-6):417-22

24. Youdim MB. Brain iron deficiency and excess; cognitive impairment and neurodegeneration with involvement of striatum and hippocampus. Neurotox Res 2008;14(1):4556.

25. Kulshreshtha A, Zacharia AJ, Jarouliya U, Bhadauriya P, Prasad GB, Bisen PS. Spirulina in health care management. Curr Pharm Biotechnol 2008;9(5):400-5.

How to cite this article: Rashmi BR. A review on spirulina (Arthrospira Platensis) for its antioxidant and neuroprotective effect. Int $J$ Comprehensive $A d v$ Pharmacol 2019;4(4):116-9. 